\title{
Convergence Analysis for a Modified SP Iterative Method
}

\author{
Fatma Öztürk Çeliker \\ Department of Mathematics, Yildiz Technical University, Davutpasa Campus, Esenler, 34220 Istanbul, Turkey \\ Correspondence should be addressed to Fatma Öztürk Çeliker; ozturkf@yildiz.edu.tr
}

Received 13 June 2014; Accepted 9 July 2014; Published 10 August 2014

Academic Editor: Syed A. Mohiuddine

Copyright (C) 2014 Fatma Öztürk Çeliker. This is an open access article distributed under the Creative Commons Attribution License, which permits unrestricted use, distribution, and reproduction in any medium, provided the original work is properly cited.

We consider a new iterative method due to Kadioglu and Yildirim (2014) for further investigation. We study convergence analysis of this iterative method when applied to class of contraction mappings. Furthermore, we give a data dependence result for fixed point of contraction mappings with the help of the new iteration method.

\section{Introduction}

Recent progress in nonlinear science reveals that iterative methods are most powerful tools which are used to approximate solutions of nonlinear problems whose solutions are inaccessible analytically. Therefore, in recent years, an intensive interest has been devoted to developing faster and more effective iterative methods for solving nonlinear problems arising from diverse branches in science and engineering.

Very recently the following iterative methods are introduced in [1] and [2], respectively:

$$
\begin{gathered}
x_{0} \in D, \\
x_{n+1}=T y_{n}, \\
y_{n}=\left(1-\alpha_{n}\right) z_{n}+\alpha_{n} T z_{n}, \\
z_{n}=\left(1-\beta_{n}\right) x_{n}+\beta_{n} T x_{n}, \quad n \in \mathbb{N}, \\
u_{0} \in D, \\
u_{n+1}=T v_{n}, \\
v_{n}=\left(1-\alpha_{n}\right) T u_{n}+\alpha_{n} T w_{n}, \\
w_{n}=\left(1-\beta_{n}\right) u_{n}+\beta_{n} T u_{n}, \quad n \in \mathbb{N},
\end{gathered}
$$

where $D$ is a nonempty convex subset of a Banach space $B, T$ is a self map of $D$, and $\left\{\alpha_{n}\right\}_{n=0}^{\infty},\left\{\beta_{n}\right\}_{n=0}^{\infty}$ are real sequences in $[0,1]$.
While the iterative method (1) fails to be named in [1], the iterative method (2) is called Picard-S iteration method in [2]. Since iterative method (1) is a special case of SP iterative method of Phuengrattana and Suantai [3], we will call it here Modified SP iterative method.

It was shown in [1] that Modified SP iterative method (1) is faster than all Picard [4], Mann [5], Ishikawa [6], and $S$ [7] iterative methods in the sense of Definitions 1 and 2 given below for the class of contraction mappings satisfying

$$
\|T x-T y\| \leq \delta\|x-y\|, \quad \delta \in(0,1), \forall x, y \in B .
$$

Using the same class of contraction mappings (3), Gürsoy and Karakaya [2] showed that Picard-S iteration method (2) is also faster than all Picard [4], Mann [5], Ishikawa [6], S [7], and some other iterative methods in the existing literature.

In this paper, we show that Modified SP iterative method converges to the fixed point of contraction mappings (3). Also, we establish an equivalence between convergence of iterative methods (1) and (2). For the sake of completness, we give a comparison result between the rate of convergences of iterative methods (1) and (2), and it thus will be shown that Picard-S iteration method is still the fastest method. Finally, a data dependence result for the fixed point of the contraction mappings (3) is proven.

The following definitions and lemmas will be needed in order to obtain the main results of this paper. 
Definition 1 (see [8]). Let $\left\{a_{n}\right\}_{n=0}^{\infty}$ and $\left\{b_{n}\right\}_{n=0}^{\infty}$ be two sequences of real numbers with limits $a$ and $b$, respectively. Suppose that

$$
\lim _{n \rightarrow \infty} \frac{\left|a_{n}-a\right|}{\left|b_{n}-b\right|}=l
$$

exists.

(i) If $l=0$, then we say that $\left\{a_{n}\right\}_{n=0}^{\infty}$ converges faster to $a$ than $\left\{b_{n}\right\}_{n=0}^{\infty}$ to $b$.

(ii) If $0<l<\infty$, then we say that $\left\{a_{n}\right\}_{n=0}^{\infty}$ and $\left\{b_{n}\right\}_{n=0}^{\infty}$ have the same rate of convergence.

Definition 2 (see [8]). Assume that for two fixed point iteration processes $\left\{u_{n}\right\}_{n=0}^{\infty}$ and $\left\{v_{n}\right\}_{n=0}^{\infty}$ both converging to the same fixed point $p$, the following error estimates,

$$
\begin{gathered}
\left\|u_{n}-p\right\| \leq a_{n} \quad \forall n \in \mathbb{N}, \\
\left\|v_{n}-p\right\| \leq b_{n} \quad \forall n \in \mathbb{N},
\end{gathered}
$$

are available where $\left\{a_{n}\right\}_{n=0}^{\infty}$ and $\left\{b_{n}\right\}_{n=0}^{\infty}$ are two sequences of positive numbers (converging to zero). If $\left\{a_{n}\right\}_{n=0}^{\infty}$ converges faster than $\left\{b_{n}\right\}_{n=0}^{\infty}$, then $\left\{u_{n}\right\}_{n=0}^{\infty}$ converges faster than $\left\{v_{n}\right\}_{n=0}^{\infty}$ to $p$.

Definition 3 (see [9]). Let $T, \widetilde{T}: B \rightarrow B$ be two operators. We say that $\widetilde{T}$ is an approximate operator of $T$ if for all $x \in B$ and for a fixed $\varepsilon>0$ we have

$$
\|T x-\widetilde{T} x\| \leq \varepsilon
$$

Lemma 4 (see $[10]$ ). Let $\left\{\sigma_{n}\right\}_{n=0}^{\infty}$ and $\left\{\rho_{n}\right\}_{n=0}^{\infty}$ be nonnegative real sequences and suppose that for all $n \geq n_{0}, \tau_{n} \in(0,1)$, $\sum_{n=1}^{\infty} \tau_{n}=\infty$, and $\rho_{n} / \tau_{n} \rightarrow 0$ as $n \rightarrow \infty$

$$
\sigma_{n+1} \leq\left(1-\tau_{n}\right) \sigma_{n}+\rho_{n}
$$

holds. Then $\lim _{n \rightarrow \infty} \sigma_{n}=0$.

Lemma 5 (see [11]). Let $\left\{\sigma_{n}\right\}_{n=0}^{\infty}$ be a nonnegative sequence such that there exists $n_{0} \in \mathbb{N}$, for all $n \geq n_{0}$; the following inequality holds. Consider

$$
\sigma_{n+1} \leq\left(1-\tau_{n}\right) \sigma_{n}+\tau_{n} \mu_{n}
$$

where $\tau_{n} \in(0,1)$, for all $n \in \mathbb{N}, \sum_{n=0}^{\infty} \tau_{n}=\infty$ and $\eta_{n} \geq 0$, $\forall n \in \mathbb{N}$. Then

$$
0 \leq \lim \sup _{n \rightarrow \infty} \sigma_{n} \leq \lim \sup _{n \rightarrow \infty} \mu_{n} .
$$

\section{Main Results}

Theorem 6. Let $D$ be a nonempty closed convex subset of a Banach space $B$ and $T: D \rightarrow D$ a contraction map satisfying condition (3). Let $\left\{x_{n}\right\}_{n=0}^{\infty}$ be an iterative sequence generated by (1) with real sequences $\left\{\alpha_{n}\right\}_{n=0}^{\infty},\left\{\beta_{n}\right\}_{n=0}^{\infty}$ in $[0,1]$ satisfying $\sum_{k=0}^{\infty} \alpha_{k}=\infty$. Then $\left\{x_{n}\right\}_{n=0}^{\infty}$ converges to a unique fixed point of $T$, say $x_{*}$.
Proof. The well-known Picard-Banach theorem guarantees the existence and uniqueness of $x_{*}$. We will show that $x_{n} \rightarrow$ $x_{*}$ as $n \rightarrow \infty$. From (3) and (1) we have

$$
\begin{aligned}
\left\|x_{n+1}-x_{*}\right\|= & \left\|T y_{n}-T x_{*}\right\| \\
\leq & \delta\left\|y_{n}-x_{*}\right\| \\
\leq & \delta\left\{\left(1-\alpha_{n}\right)\left\|z_{n}-x_{*}\right\|+\alpha_{n} \delta\left\|z_{n}-x_{*}\right\|\right\} \\
\leq & \delta\left[1-\alpha_{n}(1-\delta)\right]\left\|z_{n}-x_{*}\right\| \\
\leq & \delta\left[1-\alpha_{n}(1-\delta)\right] \\
& \times\left\{\left(1-\beta_{n}\right)\left\|x_{n}-x_{*}\right\|+\beta_{n} \delta\left\|x_{n}-x_{*}\right\|\right\} \\
\leq & \delta\left[1-\alpha_{n}(1-\delta)\right]\left[1-\beta_{n}(1-\delta)\right]\left\|x_{n}-x_{*}\right\| \\
\leq & \delta\left[1-\alpha_{n}(1-\delta)\right]\left\|x_{n}-x_{*}\right\| .
\end{aligned}
$$

By induction on the inequality (10), we derive

$$
\begin{aligned}
\left\|x_{n+1}-x_{*}\right\| & \leq\left\|x_{0}-x_{*}\right\| \delta^{n+1} \prod_{k=0}^{n}\left[1-\alpha_{k}(1-\delta)\right] \\
& \leq\left\|x_{0}-x_{*}\right\| \delta^{n+1} e^{-(1-\delta) \sum_{k=0}^{n} \alpha_{k}} .
\end{aligned}
$$

Since $\sum_{k=0}^{\infty} \alpha_{k}=\infty$, taking the limit of both sides of inequality (11) yields $\lim _{n \rightarrow \infty}\left\|x_{n}-x_{*}\right\|=0$; that is, $x_{n} \rightarrow x_{*}$ as $n \rightarrow$ $\infty$.

Theorem 7. Let $D, B$, and $T$ with fixed point $x_{*}$ be as in Theorem 6. Let $\left\{x_{n}\right\}_{n=0}^{\infty},\left\{u_{n}\right\}_{n=0}^{\infty}$ be two iterative sequences defined by (1) and (2), respectively, with real sequences $\left\{\alpha_{n}\right\}_{n=0}^{\infty}$, $\left\{\beta_{n}\right\}_{n=0}^{\infty}$ in $[0,1]$ satisfying $\sum_{k=0}^{\infty} \alpha_{k} \beta_{k}=\infty$. Then the following are equivalent:

(i) $\left\{x_{n}\right\}_{n=0}^{\infty}$ converges to $x_{*}$;

(ii) $\left\{u_{n}\right\}_{n=0}^{\infty}$ converges to $x_{*}$.

Proof. We will prove (i) $\Rightarrow$ (ii). Now by using (1), (2), and condition (3), we have

$$
\begin{aligned}
&\left\|x_{n+1}-u_{n+1}\right\|=\left\|T y_{n}-T v_{n}\right\| \\
& \leq \delta \delta y_{n}-v_{n} \| \\
&= \delta \|\left(1-\alpha_{n}\right) z_{n}+\alpha_{n} T z_{n}-\left(1-\alpha_{n}\right) T u_{n} \\
& \quad-\alpha_{n} T w_{n} \| \\
& \leq \delta\left\{\left(1-\alpha_{n}\right)\left\|z_{n}-T z_{n}\right\|+\left(1-\alpha_{n}\right) \delta\left\|z_{n}-u_{n}\right\|\right. \\
&\left.\quad+\alpha_{n} \delta\left\|z_{n}-w_{n}\right\|\right\}
\end{aligned}
$$




$$
\begin{aligned}
& \leq\left(1-\alpha_{n}\right) \delta\left\|x_{n}-u_{n}\right\| \\
& +\left(1-\alpha_{n}\right) \delta \beta_{n}\left\|T x_{n}-x_{n}\right\| \\
& +\alpha_{n} \delta\left\|z_{n}-w_{n}\right\|+\left(1-\alpha_{n}\right)\left\|z_{n}-T z_{n}\right\| \\
& \leq\left(1-\alpha_{n}\right) \delta\left\|x_{n}-u_{n}\right\|+\left(1-\alpha_{n}\right) \delta \beta_{n}\left\|T x_{n}-x_{n}\right\| \\
& +\alpha_{n} \delta\left[1-\beta_{n}(1-\delta)\right]\left\|x_{n}-u_{n}\right\| \\
& +\left(1-\alpha_{n}\right)\left\|z_{n}-T z_{n}\right\| \\
& \leq\left[1-\alpha_{n}(1-\delta)\right]\left\|x_{n}-u_{n}\right\| \\
& +\left(1-\alpha_{n}\right) \delta \beta_{n}\left\|T x_{n}-x_{n}\right\|+\left(1-\alpha_{n}\right)\left\|z_{n}-T z_{n}\right\| \text {. }
\end{aligned}
$$

Define

$$
\begin{aligned}
& \sigma_{n}:=\left\|x_{n}-u_{n}\right\|, \\
& \tau_{n}:=\alpha_{n}(1-\delta) \in(0,1), \\
& \rho_{n}:=\left(1-\alpha_{n}\right) \delta \beta_{n}\left\|x_{n}-T x_{n}\right\|+\left(1-\alpha_{n}\right)\left\|z_{n}-T z_{n}\right\| .
\end{aligned}
$$

Since $\lim _{n \rightarrow \infty}\left\|x_{n}-x_{*}\right\|=0$ and $T x_{*}=x_{*}, \lim _{n \rightarrow \infty} \| x_{n}-$ $T x_{n}\left\|=\lim _{n \rightarrow \infty}\right\| z_{n}-T z_{n} \|=0$ which implies $\rho_{n} / \tau_{n} \rightarrow 0$ as $n \rightarrow \infty$. Since also $\alpha_{n}, \beta_{n} \in[0,1]$ for all $n \in \mathbb{N}$

$$
\alpha_{n} \beta_{n}<\alpha_{n}
$$

hence the assumption $\sum_{k=0}^{\infty} \alpha_{k} \beta_{k}=\infty$ leads to

$$
\sum_{k=0}^{\infty} \alpha_{k}=\infty \text {. }
$$

Thus all conditions of Lemma 4 are fulfilled by (12), and so $\lim _{n \rightarrow \infty}\left\|x_{n}-u_{n}\right\|=0$. Since

$$
\begin{gathered}
\left\|u_{n}-x_{*}\right\| \leq\left\|x_{n}-u_{n}\right\|+\left\|x_{n}-x_{*}\right\|, \\
\lim _{n \rightarrow \infty}\left\|u_{n}-x_{*}\right\|=0 .
\end{gathered}
$$

Using the same argument as above one can easily show the implication (ii) $\Rightarrow(\mathrm{i})$; thus it is omitted here.

Theorem 8. Let $D, B$, and $T$ with fixed point $x_{*}$ be as in Theorem 6. Let $\left\{\alpha_{n}\right\}_{n=0}^{\infty},\left\{\beta_{n}\right\}_{n=0}^{\infty}$ be real sequences in $(0,1)$ satisfying

(i) $\lim _{n \rightarrow \infty} \alpha_{n}=\lim _{n \rightarrow \infty} \beta_{n}=0$.

For given $x_{0}=u_{0} \in D$, consider iterative sequences $\left\{x_{n}\right\}_{n=0}^{\infty}$ and $\left\{u_{n}\right\}_{n=0}^{\infty}$ defined by (1) and (2), respectively. Then $\left\{u_{n}\right\}_{n=0}^{\infty}$ converges to $x_{*}$ faster than $\left\{x_{n}\right\}_{n=0}^{\infty}$ does.

Proof. The following inequality comes from inequality (10) of Theorem 6:

$$
\begin{aligned}
\left\|x_{n+1}-x_{*}\right\| \leq & \left\|x_{0}-x_{*}\right\| \delta^{n+1} \\
& \times \prod_{k=0}^{n}\left[1-\alpha_{k}(1-\delta)\right]\left[1-\beta_{k}(1-\delta)\right] .
\end{aligned}
$$

The following inequality is due to ([2], inequality (2.5) of Theorem 1):

$$
\begin{aligned}
\left\|u_{n+1}-x_{*}\right\| \leq & \left\|u_{0}-x_{*}\right\| \delta^{2(n+1)} \\
& \times \prod_{k=0}^{n}\left[1-\alpha_{k} \beta_{k}(1-\delta)\right] .
\end{aligned}
$$

Define

$$
\begin{aligned}
& a_{n}:=\left\|u_{0}-x_{*}\right\| \delta^{2(n+1)} \prod_{k=0}^{n}\left[1-\alpha_{k} \beta_{k}(1-\delta)\right], \\
& b_{n}:=\left\|x_{0}-x_{*}\right\| \delta^{n+1} \prod_{k=0}^{n}\left[1-\alpha_{k}(1-\delta)\right]\left[1-\beta_{k}(1-\delta)\right] .
\end{aligned}
$$

$$
\begin{aligned}
& \text { Since } x_{0}=u_{0} \\
& \theta_{n}:=\frac{a_{n}}{b_{n}}=\frac{\delta^{n+1} \prod_{k=0}^{n}\left[1-\alpha_{k} \beta_{k}(1-\delta)\right]}{\prod_{k=0}^{n}\left[1-\alpha_{k}(1-\delta)\right]\left[1-\beta_{k}(1-\delta)\right]} .
\end{aligned}
$$

Therefore, taking into account assumption (i), we obtain

$$
\begin{aligned}
\lim _{n \rightarrow \infty} \frac{\theta_{n+1}}{\theta_{n}} & =\lim _{n \rightarrow \infty} \frac{\delta\left[1-\alpha_{n+1} \beta_{n+1}(1-\delta)\right]}{\left[1-\alpha_{n+1}(1-\delta)\right]\left[1-\beta_{n+1}(1-\delta)\right]} \\
& =\delta<1 .
\end{aligned}
$$

It thus follows from well-known ratio test that $\sum_{n=0}^{\infty} \theta_{n}<\infty$. Hence, we have $\lim _{n \rightarrow \infty} \theta_{n}=0$ which implies that $\left\{u_{n}\right\}_{n=0}^{\infty}$ is faster than $\left\{x_{n}\right\}_{n=0}^{\infty}$.

In order to support analytical proof of Theorem 8 and to illustrate the efficiency of Picard-S iteration method (2), we will use a numerical example provided by Sahu [12] for the sake of consistent comparison.

Example 9. Let $B=\mathbb{R}$ and $D=[0, \infty)$. Let $T: D \rightarrow D$ be a mapping and for all $x \in D, T x=\sqrt[3]{3 x+18} . T$ is a contraction with contractivity factor $\delta=1 / \sqrt[3]{18}$ and $x_{*}=3$; see [12]. Take $\alpha_{n}=\beta_{n}=\gamma_{n}=1 /(n+1)$ with initial value $x_{0}=1000$. Tables 1,2 , and 3 show that Picard-S iteration method (2) converges faster than all SP [3], Picard [4], Mann [5], Ishikawa [6], $S$ [7], CR [13], $S^{*}[14]$, Noor [15], and Normal-S [16] iteration methods including a new three-step iteration method due to Abbas and Nazir [17].

We are now able to establish the following data dependence result.

Theorem 10. Let $\widetilde{T}$ be an approximate operator of $T$ satisfying condition (3). Let $\left\{x_{n}\right\}_{n=0}^{\infty}$ be an iterative sequence generated by (1) for $T$ and define an iterative sequence $\left\{\widetilde{x}_{n}\right\}_{n=0}^{\infty}$ as follows:

$$
\begin{gathered}
\tilde{x}_{0} \in D, \\
\tilde{x}_{n+1}=\widetilde{T} \widetilde{y}_{n}, \\
\widetilde{y}_{n}=\left(1-\alpha_{n}\right) \widetilde{z}_{n}+\alpha_{n} \widetilde{T} \widetilde{z}_{n}, \\
\widetilde{z}_{n}=\left(1-\beta_{n}\right) \tilde{x}_{n}+\beta_{n} \tilde{T} \tilde{x}_{n}, \quad n \in \mathbb{N},
\end{gathered}
$$


TABLE 1: Comparison speed of convergence among various iteration methods.

\begin{tabular}{lcccc}
\hline Number of iterations & Picard-S & Abbas and Nazir & Modified SP & $S^{*}$ \\
\hline 1 & 3.101431265 & 3.944094141 & 3.101431265 & 3.101431265 \\
2 & 3.000970459 & 3.032885422 & 3.003472396 & 3.006099262 \\
3 & 3.000010797 & 3.001099931 & 3.000191044 & 3.000474311 \\
4 & 3.000000126 & 3.000033381 & 3.000012841 & 3.000040908 \\
5 & 3.000000001 & 3.000000928 & 3.000000964 & 3.000003733 \\
6 & 3.000000000 & 3.000000024 & 3.000000078 & 3.000000354 \\
7 & 3.000000000 & 3.000000000 & 3.000000007 & 3.000000034 \\
8 & 3.000000000 & 3.00000000000004 & 3.000000001 & 3.000000000 \\
9 & 3.000000000 & 3.000000000 & 3.000000000 & $\vdots$ \\
$\vdots$ & $\vdots$ & $\vdots$ & $\vdots$ & \\
\hline
\end{tabular}

TABLE 2: Comparison speed of convergence among various iteration methods.

\begin{tabular}{lcccc}
\hline Number of iterations & CR & Normal S & $S$ & Picard \\
\hline 1 & 3.101431265 & 3.944094141 & 3.944094141 & 14.45128320 \\
2 & 3.004853706 & 3.056995075 & 3.079213170 & 3.944094141 \\
3 & 3.000341967 & 3.004449310 & 3.007910488 & 3.101431265 \\
4 & 3.000027911 & 3.000384457 & 3.000829879 & 3.011228065 \\
5 & 3.000002459 & 3.000035123 & 3.000088928 & 3.001247045 \\
6 & 3.000000227 & 3.000003324 & 3.000009637 & 3.000015395 \\
7 & 3.000000022 & 3.000000323 & 3.000001051 & 3.000001710 \\
8 & 3.000000003 & 3.000000032 & 3.000000115 & 3.000000190 \\
9 & 3.000000000 & 3.000000003 & 3.000000013 & 3.000000021 \\
10 & 3.000000000 & 3.000000000 & 3.000000001 & 3.000000002 \\
11 & 3.000000000 & 3.000000000 & 3.000000000 & 3.000000000 \\
12 & 3.000000000 & 3.000000000 & 3.000000000 & $\vdots$ \\
$\vdots$ & $\vdots$ & $\vdots$ & $\vdots$ & \\
\hline
\end{tabular}

TABLE 3: Comparison speed of convergence among various iteration methods.

\begin{tabular}{lcccc}
\hline $\begin{array}{l}\text { Number of } \\
\text { iterations }\end{array}$ & SP & Noor & Ishikawa & Mann \\
\hline 1 & 3.101431265 & 3.101431265 & 3.944094141 & 14.45128320 \\
2 & 3.017380074 & 3.053700718 & 3.500544608 & 9.197688670 \\
3 & 3.006056041 & 3.037176288 & 3.346563527 & 7.322609407 \\
4 & 3.002849358 & 3.028678163 & 3.267333303 & 6.346715746 \\
5 & 3.001583841 & 3.023463545 & 3.218710750 & 5.744057924 \\
6 & 3.000979045 & 3.019921623 & 3.185684999 & 5.333095485 \\
7 & 3.000651430 & 3.017350921 & 3.161716071 & 5.034023149 \\
8 & 3.000457519 & 3.015395770 & 3.143487338 & 4.806124994 \\
9 & 3.000334906 & 3.013856108 & 3.129133091 & 4.626397579 \\
10 & 3.000253300 & 3.012610550 & 3.117521325 & 4.480838008 \\
11 & 3.000196722 & 3.011581068 & 3.107924338 & 4.360421594 \\
12 & 3.000156165 & 3.010715155 & 3.099852480 & 4.259063398 \\
13 & 3.000126272 & 3.009976148 & 3.092963854 & 4.172507477 \\
$\vdots$ & $\vdots$ & $\vdots$ & $\vdots$ & $\vdots$ \\
\hline
\end{tabular}

where $\left\{\alpha_{n}\right\}_{n=0}^{\infty},\left\{\beta_{n}\right\}_{n=0}^{\infty}$ are real sequences in $[0,1]$ satisfying (i) $1 / 2 \leq \alpha_{n}$, (ii) $\beta_{n} \leq \alpha_{n}$ for all $n \in \mathbb{N}$, and (iii) $\sum_{n=0}^{\infty} \alpha_{n}=\infty$. If
$T x_{*}=x_{*}$ and $\tilde{T} \tilde{x}_{*}=\tilde{x}_{*}$ such that $\tilde{x}_{n} \rightarrow \tilde{x}_{*}$ as $n \rightarrow \infty$, then we have

$$
\left\|x_{*}-\tilde{x}_{*}\right\| \leq \frac{4 \varepsilon}{1-\delta}
$$

where $\varepsilon>0$ is a fixed number and $\delta \in(0,1)$.

Proof. It follows from (1), (3), (22), and assumption (ii) that

$$
\begin{aligned}
\left\|x_{n+1}-\tilde{x}_{n+1}\right\| & =\left\|T y_{n}-T \tilde{y}_{n}+T \tilde{y}_{n}-\widetilde{T} \tilde{y}_{n}\right\| \\
\leq & \left\|T y_{n}-T \tilde{y}_{n}\right\|+\left\|T \tilde{y}_{n}-\widetilde{T} \tilde{y}_{n}\right\| \\
\leq & \delta\left\|y_{n}-\tilde{y}_{n}\right\|+\varepsilon \\
\leq & \delta\left(1-\alpha_{n}\right)\left\|z_{n}-\widetilde{z}_{n}\right\| \\
& +\delta \alpha_{n}\left\|T z_{n}-T \widetilde{z}_{n}\right\|+\delta \alpha_{n}\left\|T \widetilde{z}_{n}-\widetilde{T}_{n}\right\|+\varepsilon \\
\leq & \delta\left[1-\alpha_{n}(1-\delta)\right]\left\|z_{n}-\widetilde{z}_{n}\right\|+\delta \alpha_{n} \varepsilon+\varepsilon \\
\leq & \delta\left[1-\alpha_{n}(1-\delta)\right]\left[1-\beta_{n}(1-\delta)\right]\left\|x_{n}-\tilde{x}_{n}\right\|
\end{aligned}
$$




$$
\begin{gathered}
+\delta\left[1-\alpha_{n}(1-\delta)\right] \beta_{n} \varepsilon+\delta \alpha_{n} \varepsilon+\varepsilon \\
\leq\left[1-\alpha_{n}(1-\delta)\right]\left\|x_{n}-\tilde{x}_{n}\right\|+2 \alpha_{n} \varepsilon+\varepsilon
\end{gathered}
$$

From assumption (i) we have

$$
1 \leq 2 \alpha_{n}
$$

and thus, inequality (24) becomes

$$
\begin{aligned}
\left\|x_{n+1}-\tilde{x}_{n+1}\right\| \leq & {\left[1-\alpha_{n}(1-\delta)\right]\left\|x_{n}-\tilde{x}_{n}\right\|+4 \alpha_{n} \varepsilon } \\
\leq & {\left[1-\alpha_{n}(1-\delta)\right]\left\|x_{n}-\tilde{x}_{n}\right\| } \\
& +\alpha_{n}(1-\delta) \frac{4 \varepsilon}{1-\delta}
\end{aligned}
$$

Denote that

$$
\begin{gathered}
\sigma_{n}:=\left\|x_{n}-\tilde{x}_{n}\right\|, \quad \tau_{n}:=\alpha_{n}(1-\delta) \in(0,1), \\
\mu_{n}:=\frac{4 \varepsilon}{1-\delta} .
\end{gathered}
$$

It follows from Lemma 5 that

$$
0 \leq \limsup _{n \rightarrow \infty}\left\|x_{n}-\tilde{x}_{n}\right\| \leq \limsup _{n \rightarrow \infty} \frac{4 \varepsilon}{1-\delta} .
$$

From Theorem 6 we know that $\lim _{n \rightarrow \infty} x_{n}=x_{*}$. Thus, using this fact together with the assumption $\lim _{n \rightarrow \infty} \tilde{x}_{n}=\tilde{x}_{*}$ we obtain

$$
\left\|x_{*}-\tilde{x}_{*}\right\| \leq \frac{4 \varepsilon}{1-\delta}
$$

\section{Conflict of Interests}

The author declares that there is no conflict of interests regarding the publication of this paper.

\section{References}

[1] N. Kadioglu and I. Yildirim, "Approximating fixed points of nonexpansive mappings by a faster iteration process," http:// arxiv.org/abs/1402.

[2] F. Gürsoy and V. Karakaya, "A Picard-S hybrid type iteration method for solving a differential equation with retarded argument," http://arxiv.org/abs/1403.2546.

[3] W. Phuengrattana and S. Suantai, "On the rate of convergence of Mann, Ishikawa, Noor and SP-iterations for continuous functions on an arbitrary interval," Journal of Computational and Applied Mathematics, vol. 235, no. 9, pp. 3006-3014, 2011.

[4] E. Picard, "Mémoire sur la théorie des équations aux dérivées partielles et la méthode des approximations successives," Journal de Mathématiques pures et appliquées, vol. 6, pp. 145-210, 1890.

[5] W. R. Mann, "Mean value methods in iteration," Proceedings of the American Mathematical Society, vol. 4, pp. 506-510, 1953.
[6] S. Ishikawa, "Fixed points by a new iteration method," Proceedings of the American Mathematical Society, vol. 44, pp. 147-150, 1974.

[7] R. Agarwal, D. O Regan, and D. Sahu, "Iterative construction of fixed points of nearly asymptotically nonexpansive mappings," Journal of Nonlinear and Convex Analysis, vol. 8, pp. 61-79, 2007.

[8] V. Berinde, "Picard iteration converges faster than Mann iteration for a class of quasi-contractive operators," Fixed Point Theory and Applications, no. 2, pp. 97-105, 2004.

[9] V. Berinde, Iterative Approximation of Fixed Points, Springer, Berlin, Germany, 2007.

[10] X. Weng, "Fixed point iteration for local strictly pseudocontractive mapping," Proceedings of the American Mathematical Society, vol. 113, no. 3, pp. 727-731, 1991.

[11] Ş. M. Şoltuz and T. Grosan, "Data dependence for Ishikawa iteration when dealing with contractive-like operators," Fixed Point Theory and Applications, vol. 2008, Article ID 242916, 2008.

[12] D. R. Sahu, "Applications of the S-iteration process to constrained minimization problems and split feasibility problems," Fixed Point Theory, vol. 12, no. 1, pp. 187-204, 2011.

[13] R. Chugh, V. Kumar, and S. Kumar, "Strong convergence of a new three step iterative scheme in Banach spaces," The American Journal of Computational Mathematics, vol. 2, pp. 345-357, 2012.

[14] I. Karahan and M. Ozdemir, "A general iterative method for approximation of fixed points and their applications," Advances in Fixed Point Theory, vol. 3, no. 3, pp. 510-526, 2013.

[15] M. A. Noor, "New approximation schemes for general variational inequalities," Journal of Mathematical Analysis and Applications, vol. 251, no. 1, pp. 217-229, 2000.

[16] D. R. Sahu and A. Petruşel, "Strong convergence of iterative methods by strictly pseudocontractive mappings in Banach spaces," Nonlinear Analysis: Theory, Methods \& Applications, vol. 74, no. 17, pp. 6012-6023, 2011.

[17] M. Abbas and T. Nazir, "A new faster iteration process applied to constrained minimization and feasibility problems," Matematicki Vesnik, vol. 66, no. 2, p. 223, 2014. 


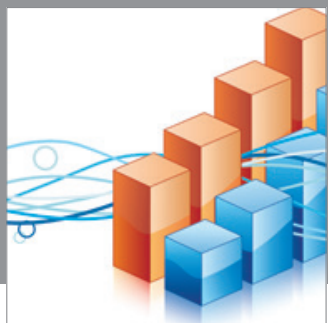

Advances in

Operations Research

mansans

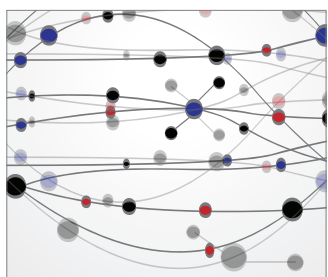

The Scientific World Journal
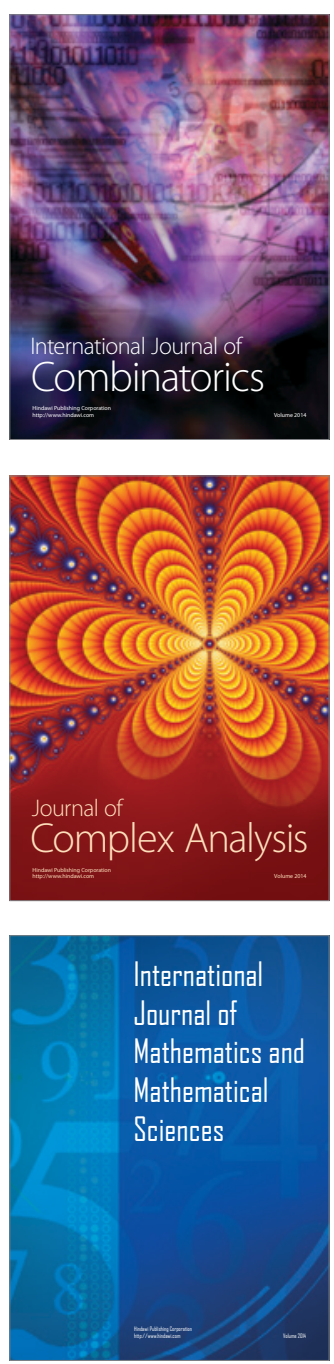
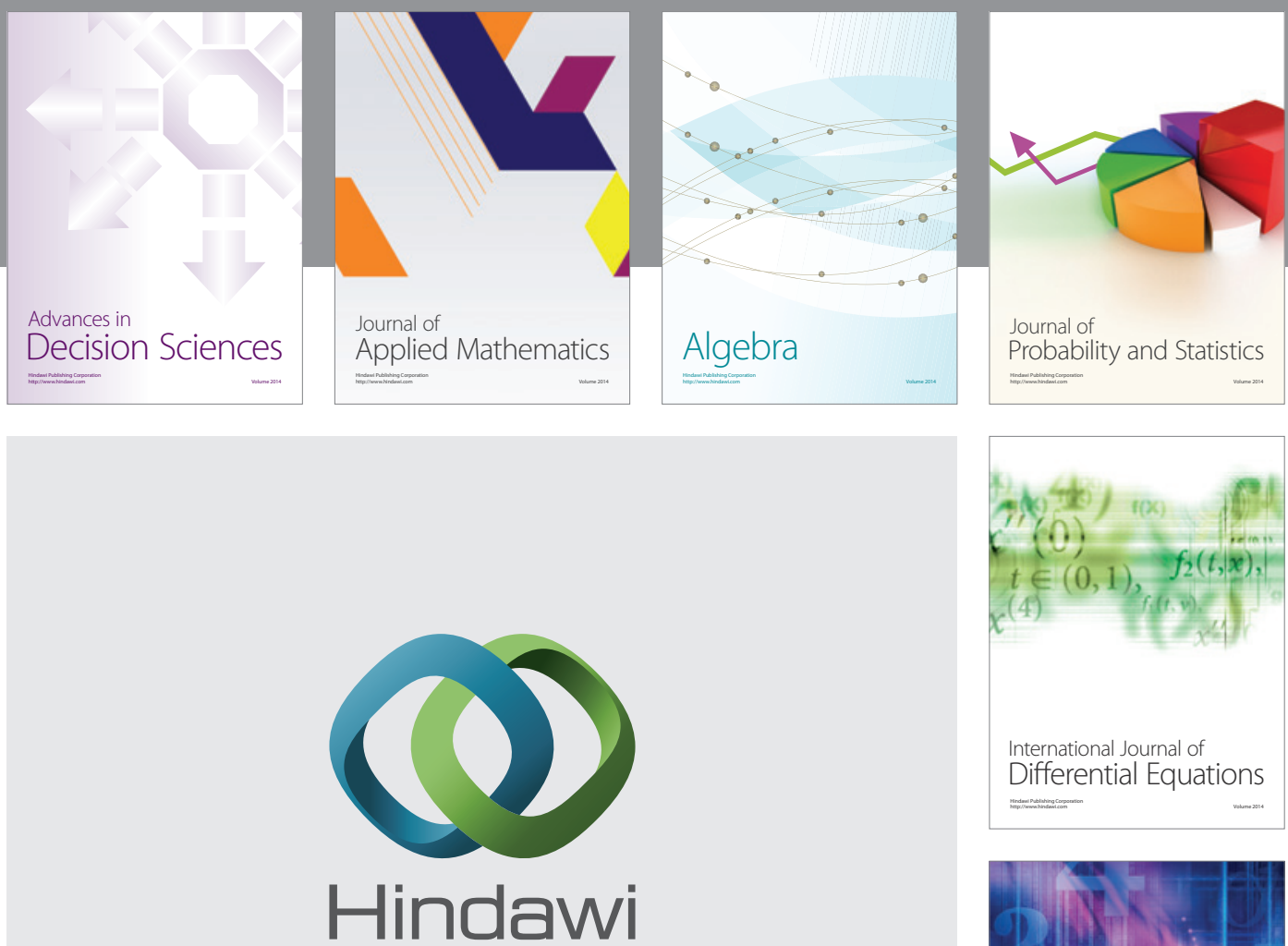

Submit your manuscripts at http://www.hindawi.com
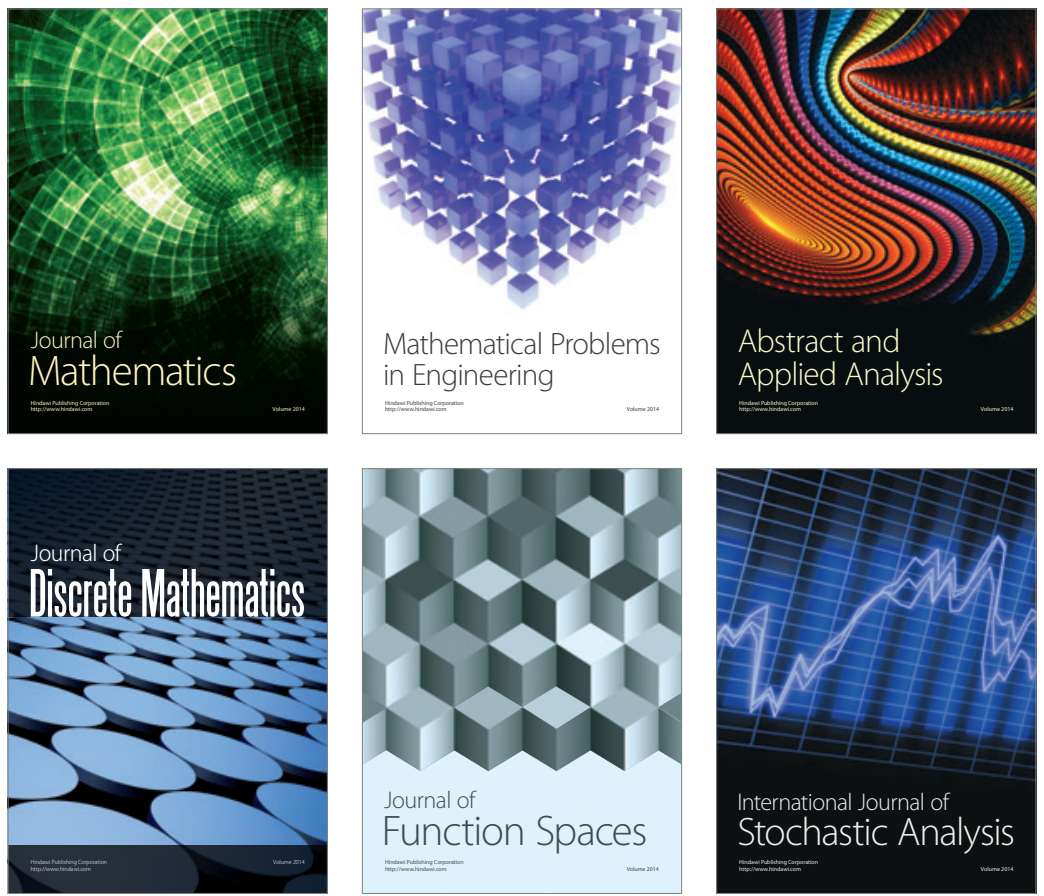

Journal of

Function Spaces

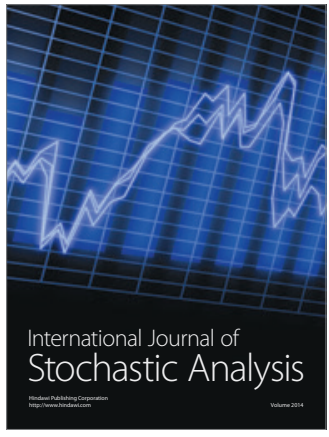

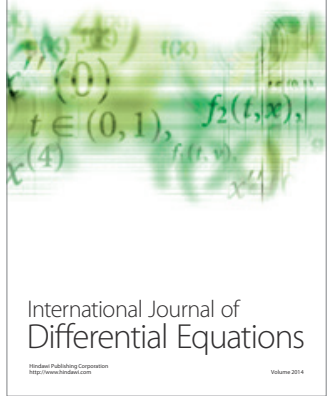
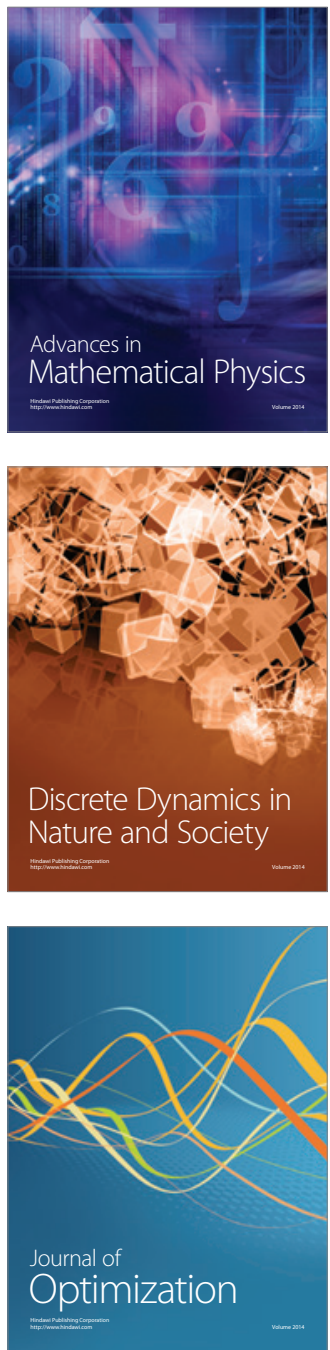